\title{
Desgaste proteico energético en pacientes con enfermedad renal crónica en estadios III a IV en Caldas, Colombia
} Protein Energy Wasting in Patients with Chronic Renal Disease in Stage III to IV Caldas (Colombia)

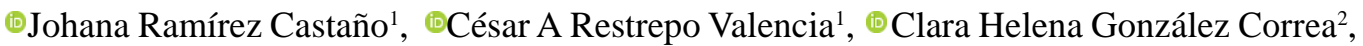 \\ -Felipe Marulanda Mejía ${ }^{1}$, 『José Arnoby Chacón Cardona ${ }^{1}$ \\ 'Departamento Clínico, Facultad Ciencias para la Salud, Universidad de Caldas, Manizales, Colombia. \\ ${ }^{2}$ Departamento de Ciencias Básicas de la salud, Facultad de Ciencias para la Salud, Universidad de Caldas, Manizales, Colombia.
}

\begin{abstract}
Resumen
Introducción: la enfermedad renal crónica (ERC) es un problema de salud mundial con una prevalencia aproximada del 7,2 \% en países desarrollados y del $10 \%$ en todo el mundo; además, es un factor independiente de morbilidad y riesgo cardiovascular que se caracteriza por la pérdida progresiva de la función renal.

Objetivo: evaluar la frecuencia de desgaste proteico energético (DPE) en pacientes con ERC en estadios III a IV.

Materiales y métodos: estudio descriptivo y de corte transversal. Se realizó una evaluación de los registros de las base de datos de la Sociedad Internacional de Nutrición y Metabolismo Renal sobre pacientes con ERC que contaran con variables sociodemográficas, bioquímicas, valoración global subjetiva (VGS) y medidas antropométricas para el diagnóstico de DPE.

Resultados: de 200 pacientes revisados en consulta externa de Nefrología, 60 cumplieron con los criterios de inclusión. El promedio de edad fue de 68,4 años, con una media de tasa de filtración glomerular (TFG) de 47,1 mL/min. Respecto a la ERC, el 61,66 \% (n=37) de los participantes fue clasificado en estadio IIIa; el 31,6 \% ( $n=19)$, en estadio IIIb, y el 6,66 \% ( $n=4)$, en estadio IV. Ninguno de los pacientes cumplió con los criterios para el DPE. La evaluación de la VGS mostró que el 53,33 \% (n=32) de los pacientes estaba en categoría VGS-A (bien nutridos), el 45 \% (n=27) en VGS-B (malnutrición moderada) y solo un paciente en VGS-C (malnutrición grave). La mayor proporción de pacientes con bajos niveles de albúmina y colesterol estuvo en pacientes con ERC en estadio IIIb, y los pacientes con índice de masa corporal <23, en estadios IIIb y IV.

Conclusión: según los criterios de la Sociedad Internacional de Nutrición y Metabolismo Renal, ningún paciente presentó DPE.

Palabras claves: deficiencia de proteína, enfermedad renal crónica, desnutrición proteica calórica (DeCS).
\end{abstract}

doi:http://dx.doi.org/10.22265/acnef.7.2.408

\begin{abstract}
Introduction: Chronic kidney disease (CKD) is a condition that is recognized as a global health problem and has an approximate prevalence of $7.2 \%$ in developed countries, and $10 \%$ in the world population, it is also an independent factor of cardiovascular morbidity and risk characterized by progressive loss of kidney function.

Objective: To evaluate the frequency of DPE in patients with CKD stages III to IV.

Methods: Descriptive, cross-sectional study. Evaluation of a database of patients with CKD, which will have sociodemographic, biochemical variables, Subjective Global Assessment (VGS), and anthropometric measures, for the diagnosis of DPE of the International Society for Nutrition and Renal Metabolism.

Results: Of 200 reviewed patients from the Nephrology outpatient clinic, 60 met the inclusion criteria. The average age was 68.4 years, with a mean glomerular filtration rate (GFR) of $47.1 \mathrm{ml} / \mathrm{min}$. Regarding CKD, 61.66\% (37) of the patients classified in stage IIIa, 31.6\% (19) in stage IIIb, and 6.66\% (4) in stage IV. None of the patients met the criteria for DPE. The VGS evaluation showed that $53.33 \%$ (32) of the patients were in the VGS A category (well nourished), 45\% (27) VGS B (moderate malnutrition) and only one patient was classified as VGS C (severe malnutrition). The highest proportion of patients with low levels of albumin and cholesterol was in patients with CKD stage IIIb, and patients with BMI less than 23 in stages IIIb and IV.

Conclusion: According to the criteria of the International Society for Renal Nutrition and Metabolism, no patient had DPE.outpatient clinic in Caldas, with CKD stages III to IV-. METHODS: Descriptive, cross-sectional study. Evaluation of a database of patients with CKD, which will have sociodemographic, biochemical variables, Subjective Global Assessment (SGA), and anthropometric measures, for the diagnosis of PEW of the International Society for Nutrition and Renal Metabolism. RESULTS: Of 200 reviewed patients from the Nephrology outpatient clinic, 60 met the inclusion criteria. The average age was 68.4 years, with a mean glomerular filtration rate (GFR) of 47.1 ml / min. Regarding CKD, 61.66\% (37) of the patients were classified in stage IIIa, 31.6\% (19) in stage $\mathrm{IIIb}$, and 6.66\% (4) in stage IV. None of the patients met the criteria for PEW. The SGA evaluation showed that 53.33\% (32) of the patients were in SGAA category (well nourished), 45\% (27) SGA B (moderate malnutrition) and only one patient was classified as SGA C (severe malnutrition). The highest proportion of patients with low albumin and cholesterol levels was in patients with CKD in stage IIIb, and patients with BMI less than 23 in stages IIIb and IV.
\end{abstract}

Conclusion: According to the criteria of the International Society for Nutrition and Renal Metabolism, no patient had PEW.

Keywords: Protein deficiency, chronic kidney disease, protein energy malnutrition (MeSH).

doi:http://dx.doi.org/10.22265/acnef.7.2.408

Citación: Ramírez Castaño J, Restrepo Valencia CA, González Correa CH, Marulanda Mejía F, Chacón Cardona JA. Desgaste proteico energético en pacientes con enfermedad renal crónica en estadios III a IV en Caldas, Colombia. Rev. Colomb. Nefrol. 2020;7(2):67-77. https://doi.org/10.22265/acnef.7.2.408 Recibido: 06.04.20, Aceptado: 03.07.20, Publicado en línea: 05.08.20

Correspondencia: Johana Ramírez Castaño, majohramirez22@gmail.com 


\section{Introducción}

$\mathrm{L}$ a enfermedad renal crónica (ERC) es una afección que se encuentra reconocida como un problema de salud mundial y que presenta una prevalencia aproximada del $7,2 \%$ en países desarrollados ${ }^{1}$, y del $10 \%$ en la población mundial ${ }^{2}$, además es un factor independiente de morbilidad y riesgo cardiovascular que se caracteriza por la pérdida progresiva de la funcionalidad del riñón ${ }^{3}$.

La ERC es un contribuyente importante a la morbimortalidad por enfermedades no transmisibles ${ }^{4}$ y, por su amplia prevalencia y su asociación con una importante morbimortalidad cardiovascular y elevados $\operatorname{costos}^{5,6}$, constituye un problema de salud emergente tanto en países desarrollados como en vía de desarrollo. Se trata de una afección progresiva y con múltiples comorbilidades, de las cuales la malnutrición es un factor relacionado importante ${ }^{7}$.

Un síndrome de cambios adversos en la nutrición y la composición corporal es altamente prevalente en pacientes con ERC, especialmente en aquellos sometidos a diálisis, y se asocia con una alta morbimortalidad $^{3}$. En específico, la prevalencia de malnutrición es elevada y aumenta a medida que disminuye la función renal ${ }^{3}$.

A medida que progresa la ERC, la acumulación de productos nitrogenados del catabolismo de proteínas dietéticas e intrínsecas puede distorsionar el gusto y el olfato y disminuir el apetito, por lo que finalmente la absorción de nutrientes gastrointestinales se vuelve anormal debido a que la uremia afecta el microbioma $y$ altera el epitelio intestinal ${ }^{8}$. Aunque la ingesta insuficiente de alimentos (desnutrición verdadera) debido al apetito deficiente y las restricciones dietéticas contribuyen a estos problemas, hay características del síndrome nutricional que no pueden explicarse solo por la desnutrición ${ }^{3}$.

Las guías de práctica clínica de nutrición para adultos con ERC en estadio III-VD y postrasplantados consideran razonable el cribado nutricional de rutina al menos cada dos años para identificar aquellos pacientes en riesgo de desgaste proteico energético $(\mathrm{DPE})^{9}$.
El concepto de DPE fue propuesto en 2007 por la Sociedad Renal Internacional de Nutrición y Metabolismo (ISRNM), quien lo definió como un estado de trastornos nutricionales y metabólicos en pacientes con ERC que se caracteriza por una pérdida simultánea y sistemática de proteína corporal y reservas energéticas que conduce a la disminución de masa muscular y grasa, y que, finalmente, lleva a caquexia ${ }^{10}$.

El panel de expertos de la ISRNM recomienda que se reconozcan cuatro categorías principales para el diagnóstico de DPE: 1) criterios bioquímicos; 2) bajo peso corporal, reducción de la grasa corporal total o pérdida de peso; 3) disminución en la masa muscular, y 4) bajo consumo de proteínas o energía ${ }^{11}$.

Estos cambios asociados con el DPE son factores de riesgo significativos para debilidad, poca capacidad de respuesta a los agentes estimulantes de la eritropoyesis, baja calidad de vida, mayor susceptibilidad a las infecciones, hospitalización y mortalidad ${ }^{10,12}$.

Las medidas usadas comúnmente en la práctica clínica para evaluar el estado nutricional incluyen el índice de masa corporal (IMC), la concentración de albúmina sérica, el recuento total de linfocitos y la circunferencia media del brazo (CMB). Adicionalmente, la valoración global subjetiva (VGS) ofrece otro enfoque para la evaluación nutricional de estos pacientes ${ }^{13,14}$.

Dado el panorama, el objetivo del presente estudio fue evaluar la frecuencia de DPE en pacientes con ERC en estadios III a IV valorados por consulta externa de nefrología en el departamento de Caldas, Colombia.

\section{Materiales y métodos}

Se realizó un estudio descriptivo transversal y cuantitativo. La población de estudio la constituyeron personas mayores de 18 años, de ambos sexos, afiliados al régimen contributivo o subsidiado de salud, con ERC en estadios III a IV (definidos según los criterios de las Guías KDIGO 2013 como anormalidades de la estructura o función renal presentes durante 3 meses) $)^{15}$ según tasa de filtración glomerular calculada por MDRD (Modification of diet in renal disease), cuyos 
datos fueron tomados de la base de datos de pacientes asistentes al servicio de consulta externa de nefrología del Departamento Clínico de la Universidad de Caldas en la ciudad de Manizales, Colombia.

Para ser incluidos, lo registros de los pacientes debían contar con información de datos sociodemográficos (área de residencia, estrato, nivel educativo, estado civil, tipo de afiliación), uso de hipolipemiantes, antihipertensivos, comorbilidades, etiología de ERC, variables bioquímicas (resultados más recientes de albúmina, creatinina, colesterol total, fósforo, potasio y triglicéridos) y clasificación según las categorías A, B y $\mathrm{C}$ de la valoración global subjetiva (VGS); para este último aspecto se tuvo en cuenta que la situación A es compatible con un estado nutricional normal, la $\mathrm{C}$ con una situación extrema de malnutrición y la B es la situación intermedia que muestra algunos datos de desnutrición ${ }^{16,17}$. De igual forma se tuvo en cuenta que se tuviera información sobre variables antropométricas (peso seco en $\mathrm{kg}$, talla en $\mathrm{cm}$, circunferencia del brazo (CB) en cm y pliegues tricipital (PCT), bicipital (PB), subescapular (PSE) y suprailíaco (PSI) en mm). A partir de esta información se calculó el IMC $\left(\mathrm{IMC}=\mathrm{kg} / \mathrm{m}^{2}\right)$, la circunferencia muscular del brazo en mm (CMB: (CB en mm) - (3.1416 x PCT en mm)), el área muscular del brazo en cm (AMB: (CMB2 en cm / (4 x П) y la grasa corporal (GC: sumatoria de 4 pliegues $\mathrm{PT}+\mathrm{PB}$
+ PSE + PSI) mediante la fórmula de Durnin and Womersley ${ }^{18}$.

Los criterios diagnósticos para DPE se evaluaron según los criterios propuestos por la Sociedad Internacional de Nutrición Renal y Metabolismo (Tabla 1). Vale la pena mencionar que se requiere cumplir con al menos tres de estos criterios para poder establecer el diagnóstico de $\mathrm{DPE}^{19}$. Se tuvieron en cuenta los valores de fósforo (no $>5 \mathrm{mg} / \mathrm{dL}$ ), potasio (valor de referencia: 3,5 - 4,5mmol/L) y triglicéridos (valor de referencia $<$ a $150 \mathrm{mg} / \mathrm{dL}$ ) ya que los trastornos de los mismos dan resultados desfavorables en la DPE?.

Se excluyeron aquellos pacientes menores de 18 años, con ERC, síndrome nefrótico, tratamiento con esteroides intercurrentes, trasplante renal, enfermedad sistémica descompensada, hospitalización en los últimos 3 meses, embarazo, enfermedad neoplásica o enfermedad gastrointestinal que hubiera afectado significativamente el estado nutricional o la concentración de albúmina sérica.

Los datos fueron consignados en una matriz de Excel y se analizaron a través del programa EPIINFO 3.5 de la Organización Mundial de la Salud. Las variables cuantitativas se reportaron como medias y

Tabla 1. Criterios diagnósticos de desgaste proteico energético para enfermedad renal crónica.

\begin{tabular}{|l|l|}
\hline \multirow{3}{*}{ Criterios bioquímicos } & $\begin{array}{l}\text { Albúmina sérica }<3,8 \mathrm{~g} / \mathrm{dL} \\
\text { Prealbúmina/transtiretina }<30 \mathrm{mg} / \mathrm{dL} \text { (solo para pacientes en diálisis) } \\
\text { Colesterol sérico }<100 \mathrm{mg} / \mathrm{dl}\end{array}$ \\
\hline Masa corporal & $\begin{array}{l}\text { Indice de masa corporal }<23 \mathrm{~kg} / \mathrm{m}^{2} \\
\text { Pérdida de peso no intencionada }>5 \% \text { del peso en } 3 \text { meses o }>10 \% \text { en } 6 \\
\text { meses } \\
\text { Porcentaje total de grasa corporal }<10 \%\end{array}$ \\
\hline Masa muscular & $\begin{array}{l}\text { Reducción del } 5 \% \text { de la masa muscular en } 3 \text { meses o del } 10 \% \text { en } 6 \\
\text { meses } \\
\text { Área de la circunferencia muscular del brazo medio reducida (reducción } \\
>10 \% \text { en relación con el percentil } 50 \text { de la población de referencia) }\end{array}$ \\
\hline Ingesta dietaria & $\begin{array}{l}\text { Ingesta proteica dietaría baja no intencional }<0,8 \mathrm{~g} / \mathrm{kg} / \text { día al menos } \\
\text { durante } 2 \text { meses en pacientes en diálisis o }<0,6 \mathrm{~g} / \mathrm{kg} / \mathrm{día} \text { en pacientes con } \\
\text { enfermedad renal crónica en estadios II-V } \\
\text { Ingesta de energía dietaría baja no intencional }<25 \mathrm{kcal} / \mathrm{kg} / \text { día durante al } \\
\text { menos } 2 \text { meses }\end{array}$ \\
\hline
\end{tabular}

Fuente: elaboración con base en Obi et al. ${ }^{10}$ 
desviaciones estándar. El estudio fue avalado por el comité de bioética de la Universidad de Caldas.

\section{Resultados}

De la base de datos de 200 pacientes, se seleccionaron 60 que cumplían los criterios de inclusión. La media de edad fue 68,4 años $( \pm 13,5)$ para toda la muestra, 70 para el sexo masculino $( \pm 11,2)$, y $66,4( \pm 16,1)$ para el femenino. El 56,6 \% $(n=34)$ de los participantes eran hombres y el 43,3\% $(n=26)$, mujeres. El $75 \%$ de los pacientes procedían de zona urbana y el 63,3\% vivían en estrato 3 a 4 (Tabla 2).

Con relación los medicamentos, se encontró un predominio de consumo de estatinas $(56,66 \%)$ y antagonistas de receptores de angiotensina II (ARA II) $(43,33 \%)$. Las comorbilidades más frecuentes fueron hipertensión arterial (HTA) con en el $80 \%$ y diabetes mellitus en el 18,3\% de los casos. La etiología hallada en la ERC fue nefroesclerosis hipertensiva en el $25 \%$ de los casos $(\mathrm{n}=15)$, otro en el $25 \%(\mathrm{n}=15)$, descono- cida en el $25 \%(\mathrm{n}=15)$, nefritis intersticial en el 11,6\% $(n=7)$ y uropatía obstructiva en el 8,3\% (n=5).

La tasa de filtración calculada con la fórmula MDR mostró una media de 47,1 mL/min ( $\pm 10,99): 49,3 \mathrm{~mL} / \mathrm{min}$ en hombres $( \pm 9,19)$ y $44,3 \mathrm{~mL} / \mathrm{min}$ en mujeres $( \pm 12,6)$. La clasificación de la ERC fue estadio IIIa en el 61,66 \% $(n=37)$ de los pacientes, estadio IIIb el 31,6 \% ( $n=19)$, y estadio IV en el 6,66 \% ( $\mathrm{n}=4)$. Las demás características demográficas se describen en la Tabla 2.

En la Tabla 3 se observan los parámetros clínicos estratificados por género. Las mujeres tuvieron menores cifras de albúmina, potasio, fósforo y triglicéridos, mientras que la media de colesterol total fue menor en hombres.

Los parámetros antropométricos se muestran en la Tabla 4; allí se evidencia, como era de esperarse, que las mujeres obtuvieron mayores valores en las variables relacionadas con la grasa corporal, mientras en los hombres se encontraron mayores valores en las variables relacionadas con la masa muscular.

Tabla 2. Características sociodemográficas y clínicas.

\begin{tabular}{|c|c|c|c|c|}
\hline \multicolumn{3}{|c|}{ Característica } & Frecuencia & Porcentaje \\
\hline \multirow{21}{*}{$\begin{array}{l}\text { Características } \\
\text { sociodemográficas }\end{array}$} & \multirow{2}{*}{ Sexo } & Masculino & 34 & 56,6 \\
\hline & & Femenino & 26 & 43,3 \\
\hline & \multirow{2}{*}{$\begin{array}{l}\text { Área de } \\
\text { residencia }\end{array}$} & Urbana & 45 & 75 \\
\hline & & Rural & 15 & 25 \\
\hline & \multirow{3}{*}{$\begin{array}{l}\text { Estrato } \\
\text { Socioeconómico }\end{array}$} & 1 y 2 & 4 & 6,6 \\
\hline & & 3 y 4 & 38 & 63,3 \\
\hline & & 5 y 6 & 18 & 29.9 \\
\hline & \multirow{6}{*}{ Educación } & Iletrado & 4 & 6,6 \\
\hline & & Primaria & 15 & 25,0 \\
\hline & & Secundaria & 12 & 20,0 \\
\hline & & Tecnología & 4 & 6,6 \\
\hline & & Profesional & 22 & 36,6 \\
\hline & & Posgrado & 3 & 5,0 \\
\hline & \multirow{5}{*}{ Estado civil } & Casado & 36 & 60,0 \\
\hline & & Soltero & 11 & 18,3 \\
\hline & & Viudo & 10 & 16,6 \\
\hline & & Unión libre & 2 & 3,3 \\
\hline & & Divorciado & 1 & 1,6 \\
\hline & \multirow{3}{*}{ Régimen } & Contributivo & 60 & 100 \\
\hline & & Subsidiado & 0 & - \\
\hline & & Vinculado & 0 & - \\
\hline
\end{tabular}

70 Desgaste proteico energético en pacientes con enfermedad renal crónica en estadios III a IV en Caldas, Colombia 


\section{Continuación Tabla 2.}

\begin{tabular}{|c|c|c|c|c|}
\hline & & Estatinas & 34 & 56,6 \\
\hline & & ARA II & 26 & 43,3 \\
\hline & & $\mathrm{BCC}$ & 16 & 26,6 \\
\hline & Medicación & BB & 13 & 21,6 \\
\hline & & IECAs & 12 & 20,0 \\
\hline & & Tiazidicos & 9 & 15,0 \\
\hline & & ARM & 7 & 11,6 \\
\hline & & Hipertensión arterial & 48 & 80,0 \\
\hline & & Diabetes mellitus & 11 & 18,3 \\
\hline & Comorbilidades & Falla cardiaca & 3 & 5,0 \\
\hline & & $\begin{array}{l}\text { Enfermedad pulmonar } \\
\text { obstructiva crónica }\end{array}$ & 2 & 3,3 \\
\hline & & $\begin{array}{l}\text { Nefroesclerosis } \\
\text { hipertensiva }\end{array}$ & 15 & 25,0 \\
\hline & & Desconocida & 15 & 25,0 \\
\hline & & Nefritis intersticial & 7 & 11,6 \\
\hline Caracteristicas & & Uropatia obstructiva & 5 & 8,3 \\
\hline & & Nefropatía por Ig A & 3 & 5,0 \\
\hline & & Hipoplasia renal & 3 & 5,0 \\
\hline & & Nefropatía diabética & 2 & 3,3 \\
\hline & Etiología ERC & $\begin{array}{l}\text { Enfermedad renal } \\
\text { poliquística }\end{array}$ & 2 & 3,3 \\
\hline & & Nefropatía por reflujo & 2 & 3,3 \\
\hline & & Pielonefritis crónica & 2 & 3,3 \\
\hline & & Nefritis lupica & 1 & 1,6 \\
\hline & & Glomerulopatia por C3 & 1 & 1,6 \\
\hline & & $\begin{array}{l}\text { Glomeruloesclerosis } \\
\text { focal y segmentaria }\end{array}$ & 1 & 1,6 \\
\hline & & $\begin{array}{l}\text { Glomerulonefritis } \\
\text { membranoproliferativa }\end{array}$ & 1 & 1,6 \\
\hline & Estadio de & IIIa & 37 & 61,6 \\
\hline & enfermedad renal & IIIb & 19 & 31,6 \\
\hline & crónica & IV & 4 & 6,6 \\
\hline
\end{tabular}

IECA: inhibidores de la enzima convertidora de angiotensina; ARA II: antagonista del receptor de angiotensina II; ARM: antagonista de receptores de mineralocorticoides; BCC: bloqueante de canales de calcio; BB: betabloqueantes; HTA.

Fuente: elaboración propia.

De otro lado, se encontró que el 53,33\% (n=32) de los pacientes se clasificó en la categoría VGS A; el $45 \%(\mathrm{n}=27)$, en la VGS B, y solo un paciente, en VGS C. La Tabla 5 muestra la relación de DPE con VGS y evidencia niveles bajos de albumina, colesterol e IMC en pacientes en categoría VGS B. La media de fósforo fue mayor en pacientes con VGS B.
Los resultados bioquímicos revelaron una mayor proporción de bajos niveles de albumina y colesterol en pacientes con ERC estadio IIIb. Asimismo, se encontró una mayor proporción de IMC bajo en pacientes con ERC estadios IIIb y IV (26,31\% y $50 \%$, respectivamente). En cuanto a la VGS, el 62,16\% $(n=23)$ de los pacientes con ERC estadio IIIa se en- 
Tabla 3. Parámetros bioquímicos según género.

\begin{tabular}{|l|c|c|c|c|c|c|}
\hline \multirow{2}{*}{\multicolumn{1}{|c|}{ Parámetro }} & \multicolumn{2}{c|}{$\begin{array}{c}\text { Total } \\
(\mathbf{n = 6 0})\end{array}$} & \multicolumn{2}{c|}{$\begin{array}{c}\text { Masculino } \\
(\mathbf{n = 3 4})\end{array}$} & \multicolumn{2}{c|}{$\begin{array}{c}\text { Femenino } \\
\text { (n=26) }\end{array}$} \\
\cline { 2 - 7 } & $\mathbf{x}$ & $\boldsymbol{\sigma}$ & $\mathbf{x}$ & $\boldsymbol{\sigma}$ & $\mathbf{x}$ & $\boldsymbol{\sigma}$ \\
\hline Albumina (g/dl) & 4,2 & 0,5 & 4,2 & 0,5 & 4,1 & 0.5 \\
\hline $\begin{array}{l}\text { Tasa de filtración glomerular } \\
\text { (ml/min) }\end{array}$ & 47,1 & 10,9 & 49,3 & 9,1 & 44,3 & 12,6 \\
\hline Creatinina (mg/dL) & 1,4 & 0.3 & 1,5 & 0.3 & 1,3 & 0,4 \\
\hline Potasio (mEq/L) & 4,4 & 0,4 & 4,4 & 0,4 & 4,3 & 0,5 \\
\hline Fosforo (mg/dL) & 3,6 & 1,4 & 3,6 & 1,8 & 3,5 & 0,9 \\
\hline Colesterol (mg/dL) & 168 & 41,6 & 165 & 36,6 & 173 & 47,7 \\
\hline Triglicéridos (mg/dL) & 149,6 & 57,5 & 159 & 55,3 & 136,8 & 59,0 \\
\hline
\end{tabular}

x: media; $\sigma$ : desviación estándar.

Fuente: elaboración propia.

Tabla 4. Parámetros antropométricos según género.

\begin{tabular}{|l|c|c|c|c|c|c|}
\hline \multirow{2}{*}{ Parámetro } & \multicolumn{2}{c|}{$\begin{array}{c}\text { Total } \\
\text { n=60 }\end{array}$} & \multicolumn{2}{c|}{$\begin{array}{c}\text { Masculino } \\
\text { n=34 }\end{array}$} & \multicolumn{2}{c|}{$\begin{array}{c}\text { Femenino } \\
\text { n=26 }\end{array}$} \\
\cline { 2 - 7 } & $\mathbf{x}$ & $\boldsymbol{\sigma}$ & $\mathbf{x}$ & $\boldsymbol{\sigma}$ & $\mathbf{x}$ & $\boldsymbol{\sigma}$ \\
\hline Peso $(\mathrm{kg})$ & 68,4 & 11,6 & 71,9 & 10,1 & 63,4 & 11,7 \\
\hline Talla $\left(\mathrm{cm}^{2}\right)$ & 160 & 10,2 & 166,7 & 6,6 & 151,4 & 7,1 \\
\hline IMC $\left(\mathrm{kg} / \mathrm{m}^{2}\right)$ & 26,6 & 3,7 & 25,9 & 3,9 & 27,6 & 3,5 \\
\hline CB $\left(\mathrm{cm}^{2}\right)$ & 31,30 & 3,4 & 30,9 & 3,9 & 31,7 & 3,6 \\
\hline CMB $\left(\mathrm{mm}^{2}\right.$ & 265,3 & 27,4 & 272,6 & 24,7 & 255,4 & 28,0 \\
\hline AMB $\left(\mathrm{cm}^{2}\right)$ & 56,3 & 11,6 & 59,6 & 10,7 & 52,1 & 11,5 \\
\hline PT $(\mathrm{mm})$ & 15,2 & 6,1 & 12,3 & 3,16 & 19,6 & 6,2 \\
\hline AGB $(\mathrm{mm})$ & 2222 & 1039 & 1742 & 529 & 2849 & 1206 \\
\hline GC $(\%)$ & 29,8 & 7,1 & 25,3 & 4,8 & 35,7 & 5,2 \\
\hline
\end{tabular}

$\mathrm{x}$ : media; $\sigma$ : desviación estándar; IMC: índice de masa corporal; $\mathrm{CB}$ : circunferencia del brazo; CMB: circunferencia muscular del brazo; AMB: área muscular del brazo; PT: pliegue tricipital; AGB: área grasa del brazo; GC: grasa corporal.

Fuente: elaboración propia.

Tabla 5. Relación entre desgaste proteico energético y valoración global subjetiva.

\begin{tabular}{|l|c|c|c|}
\hline \multicolumn{1}{|c|}{ Criterios DPE } & VGS A & VGS B & VGS C \\
\hline Albumina $<3,8$ & - & 6 & - \\
\hline Colesterol $<100$ & - & 1 & 1 \\
\hline IMC $<23 \quad$ reducción & 4 & 6 & 1 \\
\hline $\begin{array}{l}\text { CMB }>\text { del p50 } \\
>10 \% \text {. }\end{array}$ & 5 & 5 & - \\
\hline
\end{tabular}

DPE: desgaste proteico energético; VGS: valoración global subjetiva.

Fuente: elaboración propia. 
contraron clasificados como VGS A, y el 57,89\% $(n=11)$ de los pacientes con ERC estadio IIIb se encontraron clasificados como VGS B. Los demás resultados se observan en la Tabla 6 .

En la Tabla 7 se puede ver la frecuencia con que los pacientes cumplieron con algunos criterios para DPE según clasificaciones de VGS y estadio de ERC. La mayoría de pacientes clasificados en VGS A y ERC
IIIa no cumplían ningún criterio para DPE $(78,12 \%$ y $78,37 \%$ respectivamente). Un mayor porcentaje de pacientes clasificados en VGS B y C, y pacientes con ERC IIIb y IV cumplieron con uno o más criterios para DPE.

En la Tabla 8 se muestran los resultados de las variables antropométricas estratificadas por sexo y según clasificación de VGS. En hombres la media para

Tabla 6. Desgaste proteico energético y estadio de la enfermedad renal crónica.

\begin{tabular}{|c|c|c|c|c|}
\hline \multicolumn{2}{|c|}{ Criterios DPE } & ERC IIIa & ERC IIIb & ERC IV \\
\hline \multicolumn{2}{|c|}{ Albumina $<3,8$} & 3 & 3 & - \\
\hline \multicolumn{2}{|c|}{ Colesterol $<100$} & - & 1 & - \\
\hline \multicolumn{2}{|c|}{ Índice de masa corporal $<23$} & 4 & 5 & 2 \\
\hline \multicolumn{2}{|c|}{ CMB reducción $>10 \%$ del p50 } & 4 & 6 & 1 \\
\hline \multirow[t]{3}{*}{ VGS } & A & 23 & 7 & 2 \\
\hline & B & 14 & 11 & 2 \\
\hline & $\mathrm{C}$ & - & 1 & - \\
\hline
\end{tabular}

Fuente: elaboración propia.

Tabla 7. Número de criterios de desgaste proteico energético según la clasificación valoración global subjetiva.

\begin{tabular}{|l|l|c|c|c|c|}
\hline \multicolumn{2}{|c|}{ Criterios DPE/VGS-ERC } & D0 & D1 & D2 & D3 \\
\hline \multirow{5}{*}{ VGS } & A & $25(78,1 \%)$ & $5(15,6 \%)$ & $2(6,25 \%)$ & - \\
\cline { 2 - 6 } & B & $15(55,5 \%)$ & $7(25,9 \%)$ & $4(14,81 \%)$ & $1(3,7 \%)$ \\
\cline { 2 - 6 } & C & - & - & 1 & - \\
\hline \multirow{5}{*}{ ERC } & IIIa & $29(78,3 \%)$ & $5(13,5 \%)$ & $3(8,1 \%)$ & - \\
\cline { 2 - 7 } & IIIb & $9(47,3 \%)$ & $6(31,5 \%)$ & $3(15,7 \%)$ & $1(5,2 \%)$ \\
\cline { 2 - 7 } & IV & $2(50 \%)$ & $1(25,0 \%)$ & $1(25,0 \%)$ & - \\
\hline
\end{tabular}

DPE: desgaste proteico energético; VGS: valoración global subjetiva; ERC: enfermedad renal crónica. Fuente: elaboración propia.

Tabla 8. Variables antropométricas y valoración global subjetiva.

\begin{tabular}{|l|c|c|c|c|c|c|}
\hline \multicolumn{1}{|c|}{ Variable } & \multicolumn{2}{c|}{ VGS A (n=32) } & \multicolumn{2}{c|}{ VGS B (n=27) } & \multicolumn{2}{c|}{ VGS C (n=1) } \\
\hline Sexo & $\mathrm{H}(\mathrm{n}=20)$ & $\mathrm{M}(\mathrm{n}=12)$ & $\mathrm{H}(\mathrm{n}=13)$ & $\mathrm{M}(\mathrm{n}=14)$ & $\mathrm{H}(\mathrm{n}=1)$ & $\mathrm{M}(-)$ \\
\hline IMC & 26,25 & 24,29 & 23,94 & 28,81 & 21,23 & - \\
\hline CB $(\mathrm{cm} 2)$ & 32 & 29 & 28 & 32 & 26 & - \\
\hline CMB $(\mathrm{mm})$ & 278,9 & 230,4 & 246,2 & 260,1 & 247 & - \\
\hline AMB $(\mathrm{cm} 2)$ & 62,4 & 46,19 & 52,21 & 54,48 & 48,54 & - \\
\hline PT $(\mathrm{mm})$ & 12,5 & 18,2 & 10,7 & 19,7 & 4 & - \\
\hline AGB $(\mathrm{mm})$ & 1862 & 2498 & 1536 & 2972 & 507 & - \\
\hline GC \% & 27,24 & 32,82 & 21,78 & 35,98 & 12,6 & - \\
\hline
\end{tabular}

VGS: valoración global subjetiva; H: hombre; M: mujer.

Fuente: elaboración propia. 
IMC, CB, CMB, AMB, PT AGB y GC fue mayor en pacientes clasificados como VGS A, respecto a pacientes clasificados como VGS B y C. Por su parte, en mujeres fue mayor la media para reserva proteica y energética en pacientes con VGS B. No se contó con mujeres clasificadas como VGS C.

Finalmente, en la Tabla 9 se muestran los niveles de fósforo según el estadio de ERC y la clasificación de VGS; allí también se evidencia un mayor nivel de fósforo a medida que progresa la enfermedad renal y un mayor nivel de fosforo en pacientes con VGS B.

\section{Discusión}

El DPE es excepcionalmente común y está estrechamente relacionado con la morbimortalidad en pacientes con ERC, particularmente en aquellos en estadio G3b, G4 y G5 y en enfermedad renal en etapa terminal ${ }^{10}$.

En 2018, el metaanálisis de estudios observacionales de Carrero et al..$^{20}$ encontró cinco estudios que incluyeron pacientes con ERC no dependientes de diálisis en estadios III a V. Cuatro de esos estudios usaron VGS e informaron una prevalencia de DPE que varió del $11 \%$ al $18 \%$. En dicho estudio también se evidenció que los pacientes que cursaron con ERC avanzada cumplían con más criterios de diagnóstico de DPE para ERC, y con una clasificación de VGS compatible con deterioro del estado nutricional (B o C). Solo un paciente clasificado en VGS B cumplió 3 criterios de 2 categorías para DPE, bioquímica (albumina $<3,8 \mathrm{~g} / \mathrm{dL}$ $-\mathrm{CT}<100 \mathrm{mg} / \mathrm{dL}$ ) y masa corporal $(\mathrm{IMC}<23)^{20}$.

En 2017, Beddhu et al. ${ }^{21}$ encontraron que la masa corporal magra y la masa grasa fueron menores cuando los pacientes presentaban dos de los criterios no dietéticos respecto a cuando estaban presentes tres categorías, incluida la dietética. Por lo tanto, una definición modificada del síndrome DPE como la presencia de dos de tres categorías no dietéticas es quizá un mejor indicador de pérdida de proteínas o energía que una definición de tres de cuatro categorías que incluyen la categoría de la dieta. Por otra parte, la presente investigación tuvo similares limitaciones que la de Beddhu et al. ${ }^{21}$, en donde las variables dietéticas utilizadas en los criterios del DPE propuestos por la ISRNM no reflejaron la pérdida de proteínas ni de energía en las poblaciones con ERC general o moderada.

El nivel de albúmina sérica como indicador de las reservas de proteínas viscerales sugiere malnutrición o inflamación y es un fuerte predictor de mortalidad en pacientes en hemodiálisis ${ }^{7}$. Los niveles de esta proteína en los pacientes estudiados se encontraron por debajo del límite de referencia para diagnóstico de DPE en pacientes clasificados en VGS B y pacientes con estadio IIIb de la ERC.

La malnutrición resulta en una composición corporal alterada y afecta los resultados clínicos ${ }^{22}$, lo cual en el presente estudio se reflejó en los pacientes masculinos con evidencia de menor CMB y AMB que se encontraban en clasificaciones de VGS B y C.

En el presente estudio también se evidenció un mayor nivel de fósforo a medida que avanzaba la ERC debido a fallos en los mecanismos compensatorios, y en pacientes con VGS B sin llegar a niveles de hiperfosfatemia. Al respecto, Yamada et al. ${ }^{23}$ demostraron que el fósforo dietario induce inflamación sistémica y estrés oxidativo sin afectar la función renal, lo que resulta en el desarrollo de los fenotipos de

Tabla 9. Niveles de fosforo según estadio de la enfermedad renal crónica y clasificación de valoración global subjetiva.

\begin{tabular}{|l|c|c|c|}
\hline \multicolumn{1}{|c|}{ Fosforo/ERC } & ERC IIIa & ERC IIIb & ERC IV \\
\hline Fosforo & $3,54(1,75)$ & $3,61(1,07)$ & $3,9(0,68)$ \\
\hline Fosforo/VGS & VGS A & VGS B & VGS C \\
\hline Fosforo & $3,4(0,82)$ & $3,97(1,92)$ & 3,3 \\
\hline
\end{tabular}

VGS: valoración global subjetiva; ERC: enfermedad renal crónica.

Fuente: elaboración propia.

74 Desgaste proteico energético en pacientes con enfermedad renal crónica

en estadios III a IV en Caldas, Colombia

e2500-5006 Revista Colombiana de Nefrología 
DPE que incluyen pérdida de peso, hipoalbuminemia y disminución de la excreción urinaria de creatinina.

Por último, vale la pena mencionar que, según Ikizler $^{24}$, una evaluación clínicamente significativa del estado nutricional debería ser capaz de identificar y estratificar el riesgo de los pacientes con DPE; distinguir sus causas y consecuencias, así como los estados de enfermedad subyacentes, y determinar si existe un beneficio potencial de las intervenciones nutricionales.

Una limitación importante del presente estudio fue la baja representación de pacientes en estadio IV de ERC, lo que evidencia la presentación de DPE e impide una comparación con estadios menos avanzados como el IIIa y el IIIb. Asimismo, en este estudio no se cotejó el estado nutricional de los pacientes con ERC con la etiología específica de la insuficiencia renal, lo que podría influir en los resultados individuales del estado proteico calórico, como por ejemplo la diabetes, versus una enfermedad renal primaria. Adicionalmente, tampoco se realizó una anamnesis alimentaria, que es una categoría de DPE.

Es importante tener en cuenta que el hecho de que más del $50 \%$ de los pacientes estuviesen consumiendo estatinas pudo haber dificultado la interpretación de los valores de colesterol total.

\section{Conclusiones}

Los parámetros antropométricos de los pacientes clasificados como VGS A se encontraban en mejores rangos que los pacientes en VGS B y C, con una leve diferencia por sexo: mayor proporción de sobrepeso y obesidad en las mujeres clasificadas en VGS B. La mayoría de estos pacientes se encontraban en estadios intermedios de la ERC (IIIa) y fueron clasificados en VGS A.
Los pacientes con ERC estadio IIIb y IV cumplían un mayor número de criterios para DPE, pero ninguno configuró el diagnóstico de DPE. Los valores de fosforo fueron mayores en paciente con ERC III b y IV.

Es probable que aumentando el tamaño de la muestra en los estadios más avanzados de la ERC puedan encontrarse con mayor claridad los signos de DPE. Sin embargo, se resalta la necesidad de realizar siempre una valoración nutricional y una intervención temprana para evitar llegar a padecer esta condición asociada a la ERC.

Ningún marcador nutricional aislado logró reflejar de manera adecuada el estado nutricional de los pacientes con ERC.

\section{Conflicto de intereses}

Ninguno declarado por los autores.

\section{Financiación}

Ninguna declarada por los autores.

\section{Agradecimientos}

Ninguno declarado por los autores.

\section{Contribución de los autores}

Cada uno de los autores contribuyó con el diseño del trabajo de investigación, recolección de datos, actividades prácticas, elaboración de resultados y redacción del artículo. 
Rev. Colomb. Nefrol. 2020;7(2):67-77, julio-diciembre de 2020 http://www.revistanefrologia.org

http://dx.doi.org/10.22265/acnef.7.2.408

\section{Referencias}

1. Zhang QL, Rothenbacher D. Prevalence of chronic kidney disease in population-based studies?: Systematic review. BMC Public Health. 2008;8:117. https://dx.doi.org/10.1186/1471-2458-8-117.

2. Otero A, de Francisco ALM , Gayoso P, García F. Prevalence of chronic renal disease in Spain?: Results of the EPIRCE study. Nefrologia. 2010;30(1):78-86.

3. Carrero JJ, Stenvinkel P, Cuppari L, Ikizler TA, Kalantar-Zadeh K, Kaysen G, et al. Etiology of the Protein-Energy Wasting Syndrome in Chronic Kidney Disease: A Consensus Statement From the International Society of Renal Nutrition and Metabolism (ISRNM). J Ren Nutr. 2013;23(2):77-90. https://dx.doi.org/10.1053/j.jrn.2013.01.001.

4. GBD Chronic Kidney Disease Collaboration. Global, regional, and national burden of chronic kidney disease, 1990-2017: a systematic analysis for the Global Burden of Disease Study 2017. Lancet. 2020;395(10225):709-33. https://dx.doi.org/10.1016/S01406736(20)30045-3.

5. Palmer SC, Ruospo M, Campbell KL, Garcia-Larsen VG, Saglimbene V, Natale P, et al. Nutrition and dietary intake and their association with mortality and hospitalisation in adults with chronic kidney disease treated with haemodialysis: Protocol for DIET-HD, a prospective multinational cohort study. BMJ Open. 2015;5(3):e006897. https://dx.doi.org/10.1136/bmjopen-2014-006897.

6. Martínez-Castelao A, Górriz-Teruel JL, Bover-Sanjuán J, Segura-de la Morena J, Cebollada J, Escalada J, et al. Documento de consenso para la detección y manejo de la enfermedad renal crónica. Nefrologia. 2014;34(2):243-62. https://dx.doi.org/10.3265/ Nefrologia.pre2014.Feb.12455.

7. Aggarwal HK, Jain D, Chauda R, Bhatia S, Sehgal R. Assessment of Malnutrition Inflammation Score in Different Stages of Chronic Kidney Disease. Pril (Makedon Akad Nauk Umet Odd Med Nauki). 2019;39(2-3):51-61. https://dx.doi.org/10.2478/prilozi-20180042 .

8. Kalantar-Zadeh K, Fouque D. Nutritional Management of Chronic Kidney Disease. N Engl J Med. 2017;377:1765-76. https:// dx.doi.org/10.1056/NEJMra1700312.

9. National Kidney Foundation. Clinical practice guideline for nutrition in chronic kidney disease?: 2019 update. Eat right; 2019.

10. Obi Y, Qader H, Kovesdy CP, Kalantar-Zadeh K. Latest consensus and update on protein-energy wasting in chronic kidney disease. Curr Opin Clin Nutr Metab Care. 2015;18(3):254-62. https://dx.doi.org/10.1097/MCO.0000000000000171.

11. Fouque D, Kalantar-Zadeh K, Kopple J, Cano N, Chauveau P, Cuppari L, et al. A proposed nomenclature and diagnostic criteria for protein-energy wasting in acute and chronic kidney disease. Kidney Int. 2008;73(4):391-8. http://dx.doi.org/10.1038/sj.ki.5002585.

12. Namuyimbwa L, Atuheire C, Okullo J, Kalyesubula R. Prevalence and associated factors of protein-energy wasting among patients with chronic kidney disease at Mulago hospital, Kampala-Uganda: A cross-sectional study. BMC Nephrol. 2018;19(1):139. http://dx.doi.org/ 10.1186/s12882-018-0920-7.

13. Lawson JA, Lazarus R, Kelly JJ. Prevalence and prognostic significance of malnutrition in chronic renal insufficiency. J Ren Nutr. 2001;11(1):16-22. http://dx.doi.org/10.1016/s1051-2276(01)85914-8.

14. Koppler JD. National Kidney Foundation K/DOQI Clinical Practice Guidelines for Nutrition in Chronic Renal Failure. Am J Kidney Dis. 2000;37(1 Suppl 2):S66-70. http://dx.doi.org/10.1053/ajkd.2001.20748.

15. KDIGO 2012 clinical practice guideline for the evaluation and management of chronic kidney disease. Kidney Int Suppl. 2013;3(1):1-150.

16. Detsky AS, McLaughlin JR, Baker JP, Johnston N, Whittaker S, Mendelson RA, et al. What is Subjective Global Assessment of Nutritional Status? JPEN J Parenter Enter Nutr. 1987;11(1):8-13. http://dx.doi.org/10.1177/014860718701100108.

17. Makhija S, Baker J. The subjective global assessment: A review of its use in clinical practice. Nutr Clin Pract. 2008;23(4):405-9. http:/ /dx.doi.org/10.1177/0884533608321214.

18. Durnin JV, Womersley J. Body fat assessed from total body density and its estimation from skinfold thickness: measurements on 481 men and women aged from 16 to 72 Years. Br J Nutr. 1974;32(01):77-97. http://dx.doi.org/10.1079/bjn19740060.

19. Hyun YY, Lee KB, Han SH, Kim YH, Kim YS, Lee SW, et al. Nutritional status in adults with predialysis chronic kidney disease: KNOWCKD study. J Korean Med Sci. 2017;32(2):257-63. http://dx.doi.org/10.3346/jkms.2017.32.2.257.

76 Desgaste proteico energético en pacientes con enfermedad renal crónica

en estadios III a IV en Caldas, Colombia

e2500-5006 Revista Colombiana de Nefrología 


\section{Rev. Colomb. Nefrol. 2020;7(2):67-77, julio-diciembre de 2020 http://www.revistanefrologia.org}

http://dx.doi.org/10.22265/acnef.7.2.408

20. Carrero JJ, Thomas F, Nagy K, Arogundade F, Avesani CM, Chan M, et al. Global Prevalence of Protein-Energy Wasting in Kidney Disease: A Meta-analysis of Contemporary Observational Studies From the International Society of Renal Nutrition and Metabolism. J Ren Nutr. 2018;28(6):380-92. https://dx.doi.org/10.1053/j.jrn.2018.08.006.

21. Beddhu S, Chen X, Wei G, Raj D, Raphael KL, Boucher R, et al. Associations of Protein-Energy Wasting Syndrome Criteria With Body Composition and Mortality in the General and Moderate Chronic Kidney Disease Populations in the United States. Kidney Int Reports. 2017;2(3):390-9. http://dx.doi.org/10.1016/j.ekir.2017.01.002.

22. Chao CT, Tang CH, Cheng RWY, Wang MYH, Hung KY. Protein-energy wasting significantly increases healthcare utilization and costs among patients with chronic kidney disease: a propensity-score matched cohort study. Curr Med Res Opin. 2017;33(9):1705-13. http:/ /dx.doi.org/10.1080/03007995.2017.1354823.

23. Yamada S, Tokumoto M, Tatsumoto N, Taniguchi M, Noguchi H, Nakano T, et al. Phosphate overload directly induces systemic inflammation and malnutrition as well as vascular calcification in uremia. Am J Physiol Ren Physiol. 2014;306(12):1418-28. http:// dx.doi.org/10.1152/ajprenal.00633.2013.

24. Ikizler TA. A patient with CKD and poor nutritional status. Clin J Am Soc Nephrol. 2013;8(12):2174-82. http://dx.doi.org/10.2215/ CJN.04630513. 